\title{
Laser Writing of Multiscale Chiral Polymer Metamaterials
}

\author{
E. P. Furlani, ${ }^{1,2,3}$ H. S. Jee, ${ }^{2,3}$ H. S. Oh, ${ }^{3}$ A. Baev, ${ }^{3}$ and P. N. Prasad ${ }^{2,3,4}$ \\ ${ }^{1}$ Department of Chemical and Biological Engineering, University at Buffalo SUNY, Buffalo, NY 14260, USA \\ ${ }^{2}$ Department of Electrical Engineering, University at Buffalo SUNY, Buffalo, NY 14260, USA \\ ${ }^{3}$ Institute for Lasers, Photonics and Biophotonics, University at Buffalo SUNY, Buffalo, NY 14260, USA \\ ${ }^{4}$ Department of Chemistry, University at Buffalo SUNY, Buffalo, NY 14260, USA
}

Correspondence should be addressed to E. P. Furlani, efurlani@buffalo.edu

Received 1 July 2012; Revised 31 July 2012; Accepted 31 July 2012

Academic Editor: Ivan D. Rukhlenko

Copyright ( $) 2012$ E. P. Furlani et al. This is an open access article distributed under the Creative Commons Attribution License, which permits unrestricted use, distribution, and reproduction in any medium, provided the original work is properly cited.

\begin{abstract}
A new approach to metamaterials is presented that involves laser-based patterning of novel chiral polymer media, wherein chirality is realized at two distinct length scales, intrinsically at the molecular level and geometrically at a length scale on the order of the wavelength of the incident field. In this approach, femtosecond-pulsed laser-induced two-photon lithography (TPL) is used to pattern a photoresist-chiral polymer mixture into planar chiral shapes. Enhanced bulk chirality can be realized by tuning the wavelength-dependent chiral response at both the molecular and geometric level to ensure an overlap of their respective spectra. The approach is demonstrated via the fabrication of a metamaterial consisting of a two-dimensional array of chiral polymer-based L-structures. The fabrication process is described and modeling is performed to demonstrate the distinction between molecular and planar geometric-based chirality and the effects of the enhanced multiscale chirality on the optical response of such media. This new approach to metamaterials holds promise for the development of tunable, polymer-based optical metamaterials with low loss.
\end{abstract}

\section{Introduction}

Metamaterials are artificial electromagnetic materials with constituent elements that are subwavelength in size relative to the incident field and engineered to produce bulk electromagnetic properties not readily found in nature. The interest in these materials has grown steadily since the seminal theoretical work by Pendry [1] and subsequent experimental work by Shelby et al. [2]. Various interesting and exotic phenomena can be realized using metamaterials including the reverse Vavilov-Cherenkov effect [3], negative refraction $[1,2]$, sub-diffraction-limit imaging $[1,4-6]$, the reverse Doppler effect, slow light [7-9], and cloaking [10-12]. Applications of metamaterials span the electromagnetic (EM) spectrum with potential transformative impact in fields such as imaging (superlens) [1, 13], data storage [14], optical switching [15], electromagnetic shielding, and stealth technology [16-18].

To date, the majority of research on metamaterials has focused on developing negative index materials (NIMs) as first proposed by Veselago [3]. Initially, these were designed to have simultaneous negative values of permittivity, $\varepsilon$, and permeability, $\mu$. To achieve this, many of the early NIMs operating at $\mathrm{GHz}$ and $\mathrm{THz}$ frequencies made use of an electromagnetic resonance involving subwavelength metallic structures such as split-ring resonators (SRR) [19] and pairs of cut wires (PCW) $[20,21]$. However, it was found that this approach has drawbacks. The reliance on resonance leads to NIM behavior with limited bandwidth, and the use of metallic structures results in relatively high loss. As the field advanced, other metamaterial constructs such as fishnet structures $[22,23]$ emerged and were shown to be superior to SRR and PCW designs in terms of a lower loss and ease in achieving overlap between electric and magnetic resonances. Nevertheless, the desire for practical metamaterials with wide bandwidth and low loss persists. In addition to performance limitations, there are issues associated with the fabrication of metamaterials, especially for use at optical frequencies. To date, the majority of such materials for $\mathrm{THz}$ applications have been fabricated using "top-down" techniques such as electron-beam lithography (EBL) or focusedion beam (FIB) milling [23]. While these methods provide an 
adequate nanometer resolution for tailoring subwavelength constituent elements, they tend to be limited to the fabrication of $2 \mathrm{D}$ planar materials on rigid substrates at low throughput and with high cost. Currently, there is intense interest and effort towards the development of fabrication methods and materials that overcome the aforementioned limitations.

In this paper, we introduce a new laser-based chiral chemical approach to metamaterials that overcomes some of the limitations of more conventional approaches. Our approach involves the use of a pulsed laser to pattern novel chiral polymer materials that exhibit chirality at two distinct length scales, intrinsically at the molecular level and geometrically at a length scale on the order of the wavelength of the incident field. Specifically, we use femtosecondpulsed laser-induced two-photon initiated polymerization of a photoresist, which is premixed with a chiral polymer, to write patterned arrays of chiral polymer-based 2D planar chiral structures. Thus, this fabricated metamaterial exhibits chirality due to both its molecular structure and geometric shape. The idea is to obtain enhanced bulk chirality by tuning the wavelength-dependent chiral response at both the molecular and geometric level so as to ensure an overlap in their respective spectra.

Chiral metamaterials offer an alternate route to NIMs as compared to the more conventional metallic-structurebased resonance approach. The chirality of a material can be characterized in terms of a chirality parameter $\kappa$. If $\kappa$ is sufficiently large, a bulk negative index can be realized within the material. Specifically, in a chiral material, negative refraction will occur at one of the eigen (circular) polarization states of the incident field if $\kappa$ is larger than the square root of the product of real parts of permittivity and permeability, that is, when

$$
\kappa>\sqrt{\varepsilon^{\prime} \cdot \mu^{\prime}}
$$

where the refractive index is given by

$$
n=\sqrt{\varepsilon^{\prime} \cdot \mu^{\prime}}-\kappa
$$

and $\varepsilon^{\prime}$ and $\mu^{\prime}$ are the relative values of the real parts of the permittivity and permeability, respectively. Thus, in the chiral approach to NIMs, a negative index can potentially be achieved with a wider bandwidth and lower loss as there is no need to introduce lossy resonant metallic constituents. Our strategy is to use a laser to fabricate materials with enhanced multiscale chirality (sufficiently large $\kappa$ ) in order to obtain polymer-based NIMs without resorting to top-down fabrication or resonant metallic structures as is common in conventional approaches to metamaterials. In the following, we report of the progress towards this end by describing the synthesis and demonstrating the fabrication of novel 2D planar media possessing multiscale chirality. We also present modeling results that demonstrate the effects of multiscale chirality on the optical response (optical rotation) of such media.

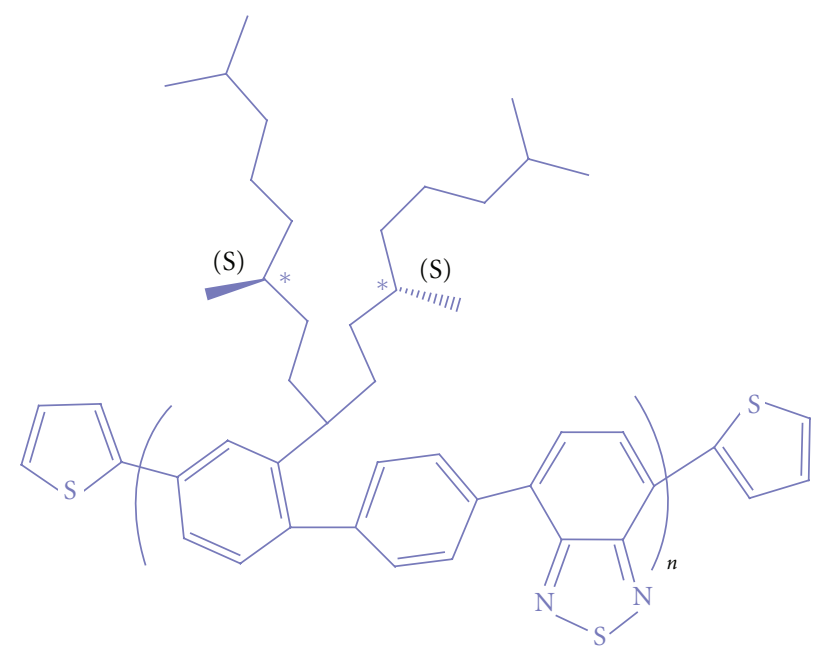

FIgURE 1: Chemical structure of PFBT polymer.

\section{Fabrication}

The fabrication of our multiscale chiral media begins with the formation of a photoresist-chiral polymer mixture consisting of SU-8 2025 from Microchem and Chiral Poly(fluorene-alt-benzothiadiazole) (PFBT) in a mixture ratio of $35: 1$. The PFBT was synthesized using palladium-catalyzed Suzuki polycondensation as the final step and purified with a Soxhlet extraction. We introduced chirality in PFBT of the fluorene-based monomer modified with (S)-3,7dimethyloctyl substituents at the 9 positions as shown in Figure 1 and described in [24]. The photoresist-chiral polymer mixture is stirred for 24 hours and then spin-coated at $1000 \mathrm{rpm}$ on a glass substrate. After spin coating, the sample is baked for $30 \mathrm{~min}$ at $95^{\circ} \mathrm{C}$ to evaporate the solvent before performing lithograph patterning. The circular dichroism (CD) (differential absorption of left and right circularly polarized light) and absorption spectra of both the pure PFBT polymer and SU-8/PFBT blend are shown in Figure 2. Note that the SU-8/PFBT exhibits a 68 -fold increase in optical activity at $\lambda=500 \mathrm{~nm}$. Presently, the reason for the enhanced optical activity is not fully understood and is the subject of continued investigation by our group. The chirality parameters for the PFBT polymer and the SU-8/PFBT are on the order of $\kappa \sim 10^{-5}$ and $2 \times 10^{-3}$, respectively.

In order to obtain multiscale chirality, we pattern the chiral SU-8/PFBT film into a planar array of L-structures using two-photon photolithography (TPL). We use a near-IR $(800 \mathrm{~nm})$ writing wavelength and a schematic of the writing set up is shown in Figure 3(a). In this system, a piezo stage moves the sample relative to a fixed laser beam and a desired pattern is produced within the sample by controlling the motion of the stage while exposing the sample. Once the sample is patterned, it is developed with Propylene Glycol Methyl Ether Acetate for 3 hours, which removes the unexposed material leaving only the patterned media. We have recently reported the use of this method for writing novel subwavelength polymer-based planar plasmonic metamaterials, which were prepared using SU-8 with a high gold 


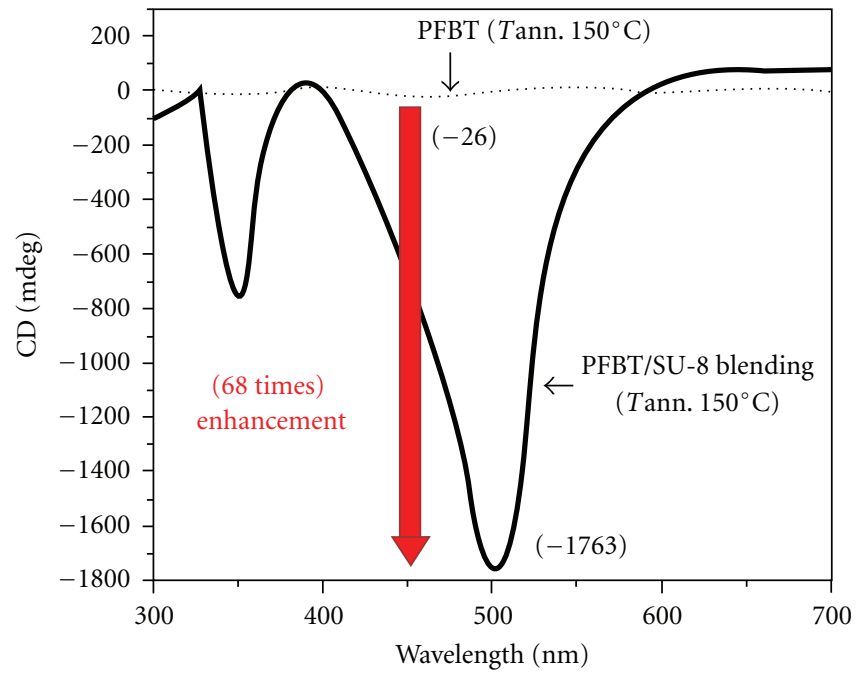

(a)

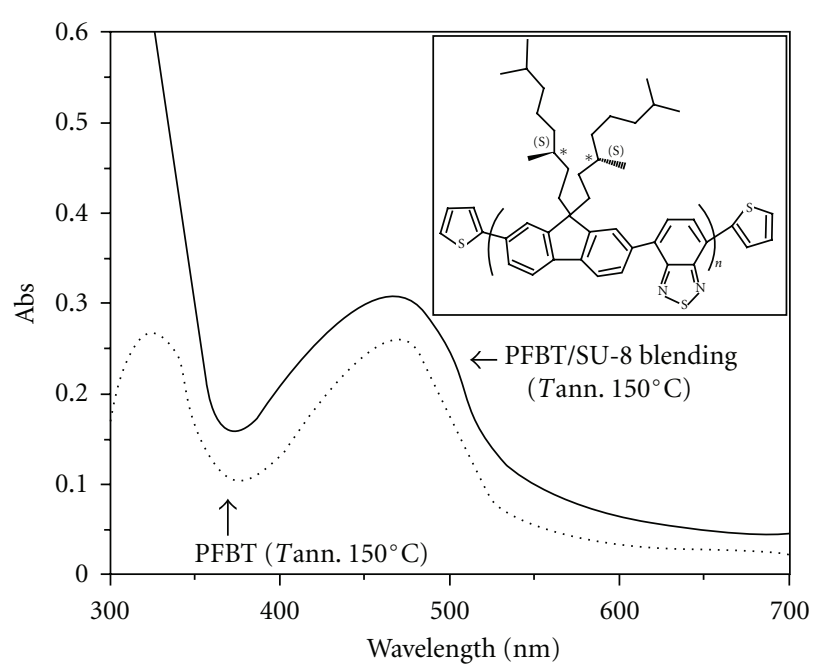

(b)

Figure 2: CD (a) and UV-vis absorption (b) spectra of (dots) annealed pure PFBT film; (solid) PFBT/SU-8 blending, 1/30 mass ratio film spin-coated from $20 \%$ solid content solution, after prebaking and annealing.

precursor (metallic salt) loading $[25,26]$. Specifically, we used a femtosecond-pulsed laser to induce two-photon initiated in situ reduction of the metal salt and simultaneous polymerization of the SU8. Gold nanoparticles are formed during the writing process, which renders a plasmonic functionality in the written structures [26]. Figure 3(b) shows examples of different written structures, which demonstrate the versatility of the writing process. The present effort on patterning chiral SU-8/PFBT film is an extension of this prior work on plasmonic structures.

A 2D array of planar L-structures fabricated using this method is shown in Figure 4. Note that while the L geometry is not chiral in a $3 \mathrm{D}$ sense, it is considered chiral in a $2 \mathrm{D}$ planar sense because it cannot be mapped onto its mirror image using a sequence of rotations or translations confined to the plane; that is, it needs to be lifted out of the plane and rotated to achieve this. Thus, based on geometric considerations alone, that is, ignoring the chirality of the SU-8/PFBT, the fabricated material is not chiral in 3D; for example, it would not rotate polarization of waves propagating in the plane of L-structures, rather it is bianisotropic. However, in addition to the planar geometric chirality, the L-structures possess intrinsic chirality due to the constituent SU-8/PFBT. Thus, the material shown in Figure 4 represents a 2D planar metamaterial with multiscale chirality. In principle, this process can be used to fabricate bulk enhanced 3D chiral metamaterials layer-by-layer, wherein a stacked sequence of carefully chosen $2 \mathrm{D}$ patterns combine to produce a $3 \mathrm{D}$ unit cell chiral geometry, an approach that we are currently pursuing.

\section{Theory and Simulation}

From a theoretical perspective, a periodic array of planar chiral L-structure elements, such as shown in Figure 4, will exhibit circular birefringence in the visible and near-infrared regions of the spectrum when their linear dimensions are close to these wavelengths $[27,28]$. In principle, the molecular chirality of the constituent polymer can be tuned to occur at these wavelengths as well, allowing for the development of materials with enhanced multiscale chirality as described above.

We use computational electrodynamics to model the electromagnetic response of chiral polymer-based chiral Lstructures as shown in Figure 4. However, to facilitate the computational analysis, we use submicron geometric dimensions, that is, slightly smaller than those shown in Figure 4. In order to take into account the overlap of $2 \mathrm{D}$ geometric chirality with molecular chirality $[24,29]$ we modify Maxwell's equations to include natural optical activity. This emerges when the spatial dispersion of a medium is considered along with the time dispersion in the linear relation between the applied electric field and the medium's displacement vector [30]. In case of relatively weak spatial dispersion, the dielectric permittivity tensor, $\varepsilon_{i j}(\omega, \mathbf{k})$, can be expanded in a series over powers of the wave vector. The coefficient of the first-order term can be related to the macroscopic chirality parameter of the medium after some algebraic operations. The 0th-order term represents the usual local dielectric permittivity tensor. In the case of an isotropic medium, consisting of a randomly oriented collection of chiral molecules, both the 0th-and 1st-order coefficients become averaged.

To compute the rotation of polarization of an incident field we employed the Finite-Element-(FE) based COMSOL multiphysics RF solver using a time-harmonic approximation (http://www.comcol.com/). The computational domain for the L-Structure is shown in Figure 5. In this model, the polymeric L-structure resides on a glass substrate with a refractive index of 1.6. The base of the unit cell is $1050 \mathrm{~nm}$ by $750 \mathrm{~nm}$, and its height is 2 microns. The dimensions of the L-shape are as follows: $750 \mathrm{~nm}$ in length, $450 \mathrm{~nm}$ at the base, the width of both branches is $150 \mathrm{~nm}$, and the thickness 


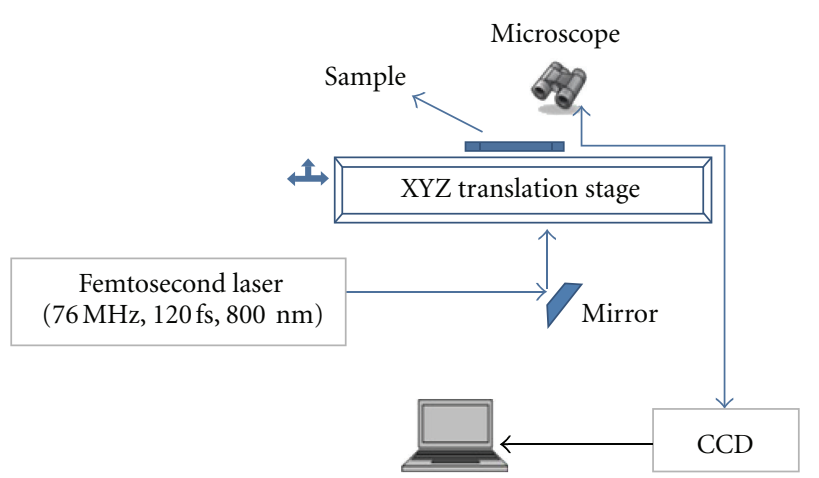

(a)

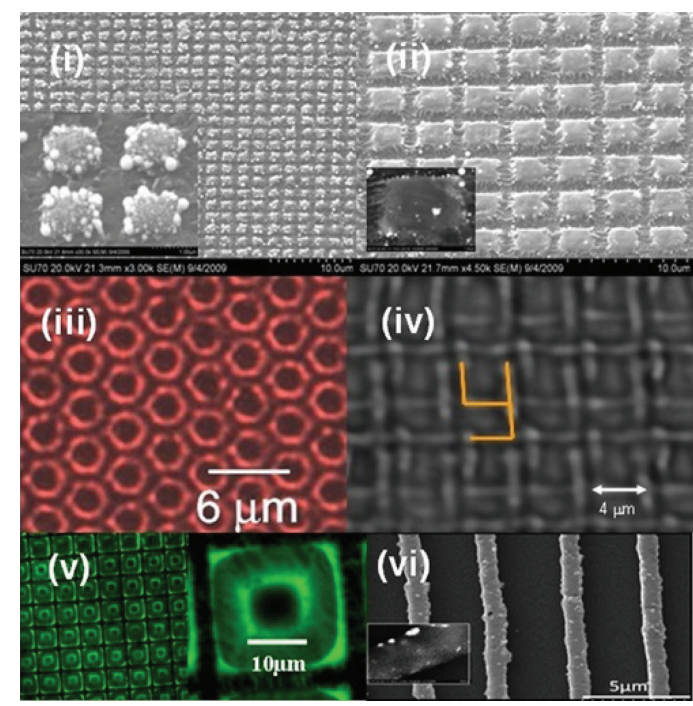

(b)

FIGURE 3: Laser-based TPL fabrication of metamaterials: (a) schematic of the TPL laser writing system, (b) SEM images of fabricated polymer-based plasmonic structures (i) cauliflowers; (ii) blocks; confocal microscope image of (iii) hexagonal ring structures; (iv) planar chiral Y structures with highlighted geometry; (v) donuts; SEM image of (vi) grating structure (adapted from [25]).

is $500 \mathrm{~nm}$. Note that while these dimensions are slightly smaller than the fabricated structures shown in Figure 4, the analysis presented here is scalable as long as the molecular chirality can be tuned to overlap the structural chirality. The isotropic refractive index of the modeled L-structure is 1.4. Scattering (low reflection) boundary conditions with Perfectly Matching Layers (PML) are applied at the top and bottom of the solution space as described in [31, 32]. Double periodic boundaries are imposed on the side boundaries to account for the fact that this structure is one element of a $2 \mathrm{D}$ array, that is, the periodic conditions account for the presence of the other elements, even though they do not appear in the model. The incident (input) field is generated by a time-harmonic surface current source positioned in the $x-y$ plane $[31,32]$. The magnitude of the surface current is chosen to provide an $x$-polarized plane wave with a field magnitude of $E_{x}=2 \times 10^{6} \mathrm{~V} / \mathrm{m}[31-33]$.

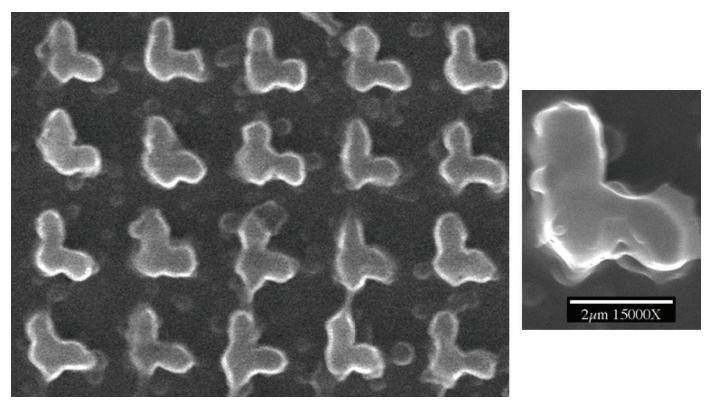

FIgure 4: SEM of fabricated chiral polymer-based chiral Lstructures.

The intrinsic chirality of the L-structure is introduced via the bulk chirality parameter, $\kappa$, which is related to macroscopic optical rotation of the medium. Here, we are not concerned with the microscopic origin of chirality [29]. Instead, we model the frequency dispersion of chirality parameter with a damped Lorentzian centered at a certain resonant wavelength. Specifically, the permittivity tensor for our analysis is of the following diagonal form:

$$
\varepsilon_{i i}(\omega)=n^{2}+\kappa^{\prime}(\omega) \sqrt{\frac{\mu_{0}}{\varepsilon_{0}}} \frac{H_{i i}}{E_{i i}},
$$

where

$$
\kappa^{\prime}(\omega)=\frac{\kappa_{0}}{\left(\omega-\omega_{0}\right)+i \Gamma}
$$

and $n$ is homogeneous refractive index, $\omega_{0}$ is the resonant frequency of molecular optical activity, and $\Gamma$ is the damping rate of corresponding molecular resonance. In our simulations the damping rate is set to $10^{13} \mathrm{sec}^{-1}$ and $\kappa_{0}$ to $10^{12} \mathrm{sec}^{-1}$. It should be noted that the specific form of permittivity tensor (3) results in the corresponding dispersion relation and an equation for the square of the effective refractive index of the medium. Equation (2) is then derived via Taylor expansion over the small parameter, assuming the smallness of chirality parameter, $\kappa^{\prime}(\omega)$. Therefore $\kappa$ of (2) and $\kappa^{\prime}(\omega)$ of (3) are related through a simple formula: $\kappa^{\prime}(\omega)=2 * n * \kappa$.

It is instructive to note that the resonance is a molecular resonance and basically determined by the microscopic electronic structure of constituent chiral molecules. Significantly, the resonance can be tuned in a relatively broad range from UV to near IR for a class of $\pi$-conjugated donor-acceptor molecules depending on the donor and acceptor strength and conjugation length. For example, the first absorption band of the PFBT molecule was tuned to approximately $500 \mathrm{~nm}$ to overlap with the plasmon resonance of gold nanoparticles in our previous work [30]. Shifting the molecular resonance to the infrared region, with a simultaneous decrease of the linear dimensions of the L-structure, results in an overlap of resonant features and an effective increase of the degree of optical rotation. The peak absolute value of chirality parameter, $\left|\kappa_{\max }^{\prime}\right|$, was set to 0.1 in our simulations. This value is a projected maximum that can be readily achieved with combined plasmonic [24], excitonic [34], and structural 


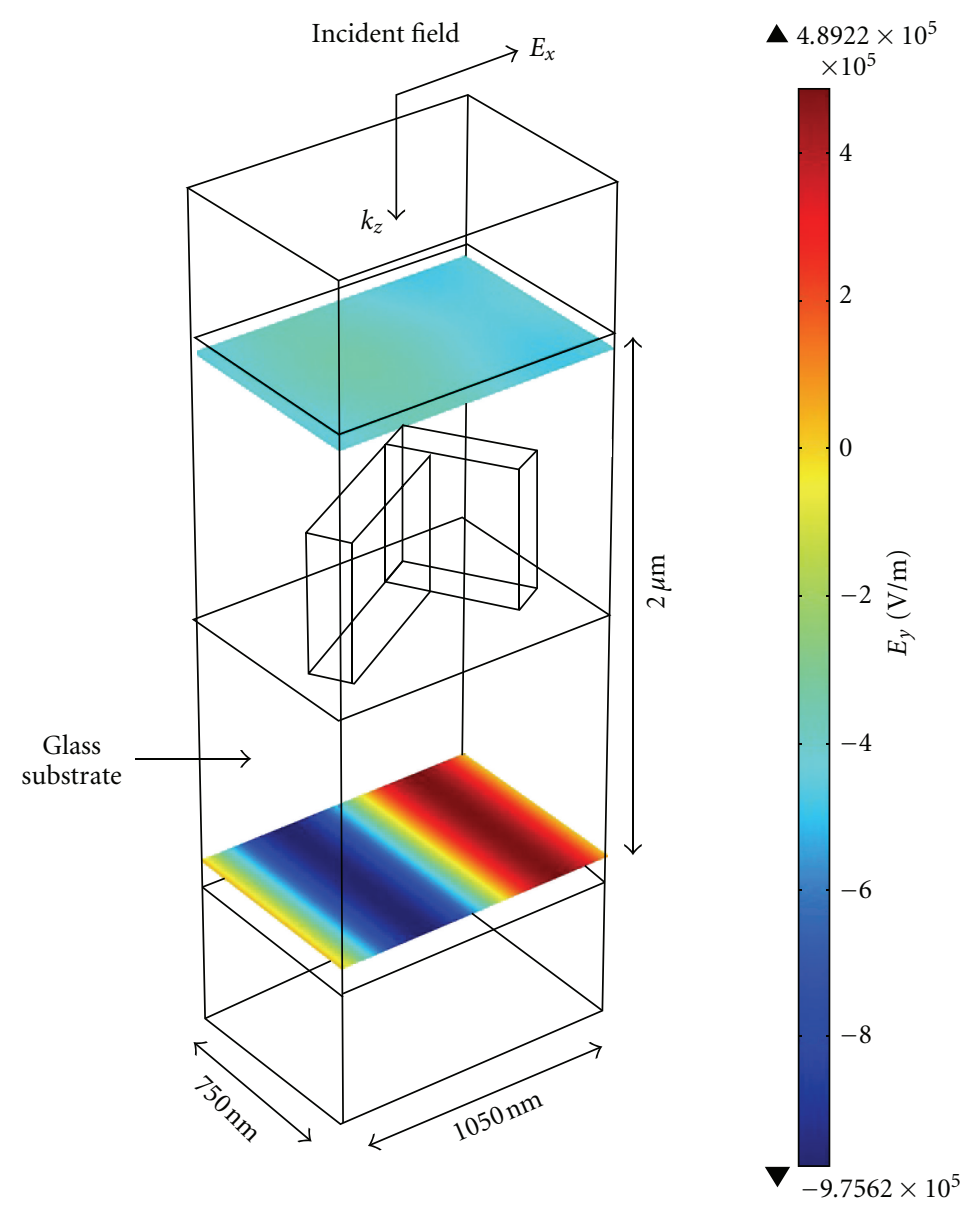

FIGURE 5: Computational domain for the planar chiral L-structure EM analysis.

enhancement [35] of chirality. Even higher values of chirality parameter can be expected from combinations of natural optical activity with (i) a magneto-optical effect enhanced by magnetoplasmonic nanoparticles, (ii) further modifications of chiral molecular building blocks, and/or (iii) chiral aggregation in thick films. The optical rotation of an Lstructure with molecular chirality is compared to one with just spatial chirality in Figure 5 and the discussion thereof.

To make sure the observed optical rotation is due to actual chirality of the L-shapes and not a computational artifact or accidental diffraction pattern we compared the EM transmission of a dielectric sphere to that of sphere made from chiral material (Figure 6). The dielectric sphere should show no optical activity as the geometry it is not chiral. As shown in Figure 7, and discussed below, the polarization state of the incident light was preserved in the former case as expected. However, the sphere consisting of chiral molecules, and therefore possessing a bulk chirality parameter, showed a chiral signature.

The results of our simulations of optical rotation are presented in Figure 7. The angle is computed with respect to the initial polarization of the incoming field. First, note that the L-structure without molecular chirality rotates the polarization in a broad range. Rotation in this case is solely determined by the shape and dimensions of the scattering object and is not resonant in nature. However, the Lstructure made of chiral material shows a narrow resonance on top of the broad rotation curve. Similarly, in the case of the spherical geometry one can see that there is no rotation of polarization in the case of the dielectric sphere. However, the chiral sphere reveals rotation due to the chiral nature of its material: the chiral signature is centered at the wavelength of the molecular resonance. This analysis demonstrates the distinction between molecular and geometric-based chirality and the effects of the enhanced multiscale chirality on the optical response of the media.

\section{Conclusions}

We have introduced a novel approach to metamaterials that combines the use of ultrafast pulsed laser-based fabrication with chiral chemistry to produce a new class of polymeric metamaterials that have enhanced bulk chirality. The enhanced chirality is achieved by tuning the wavelengthdependent chiral response of the media at both the molecular and geometric level so as to ensure an overlap of 


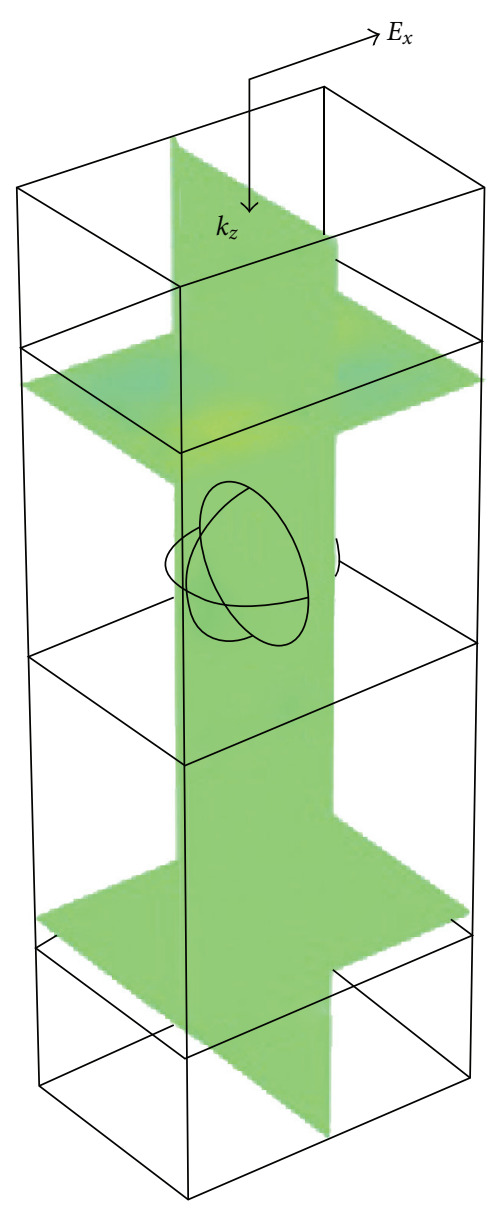

(a)
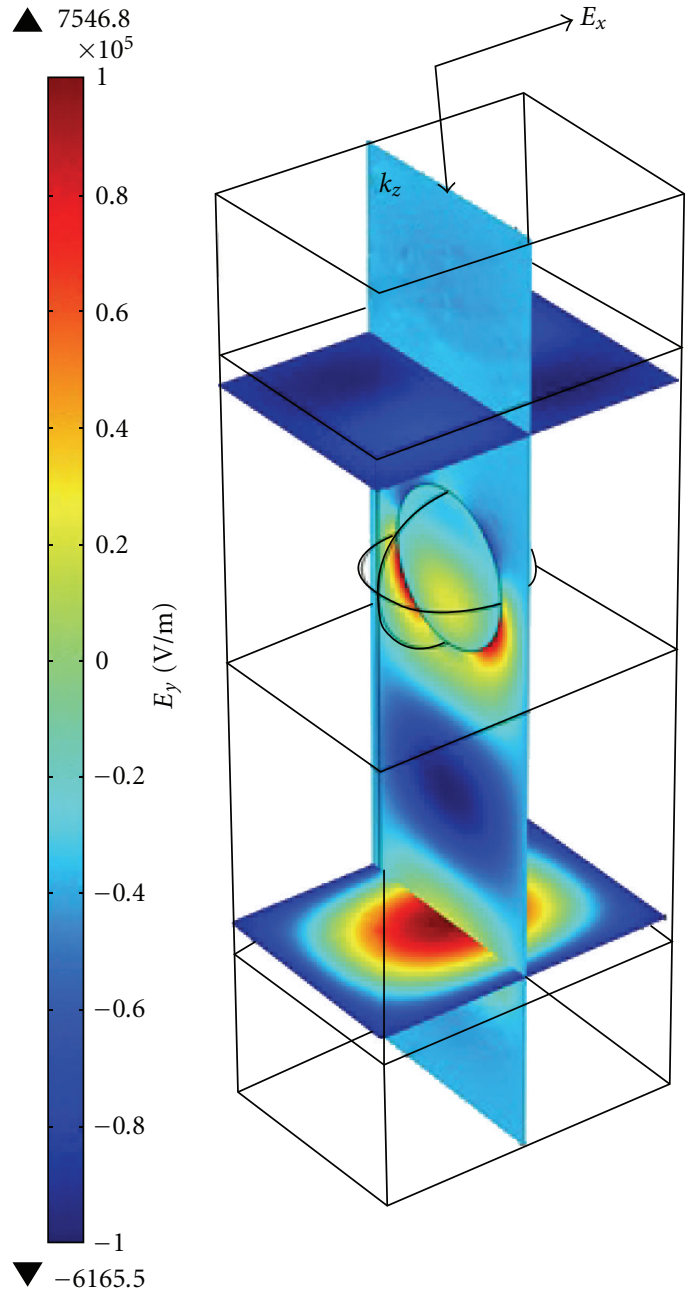

(b)

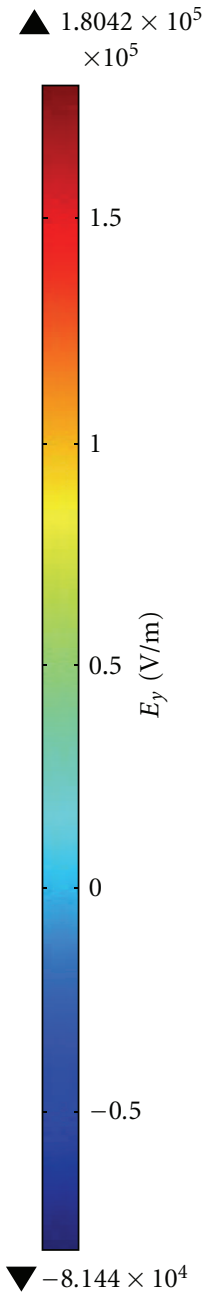

FIGURE 6: Simulated EM transmission ( $y$-component of the E-field is plotted): (a) dielectric sphere without intrinsic molecular chirality; (b) sphere with intrinsic molecular chirality.

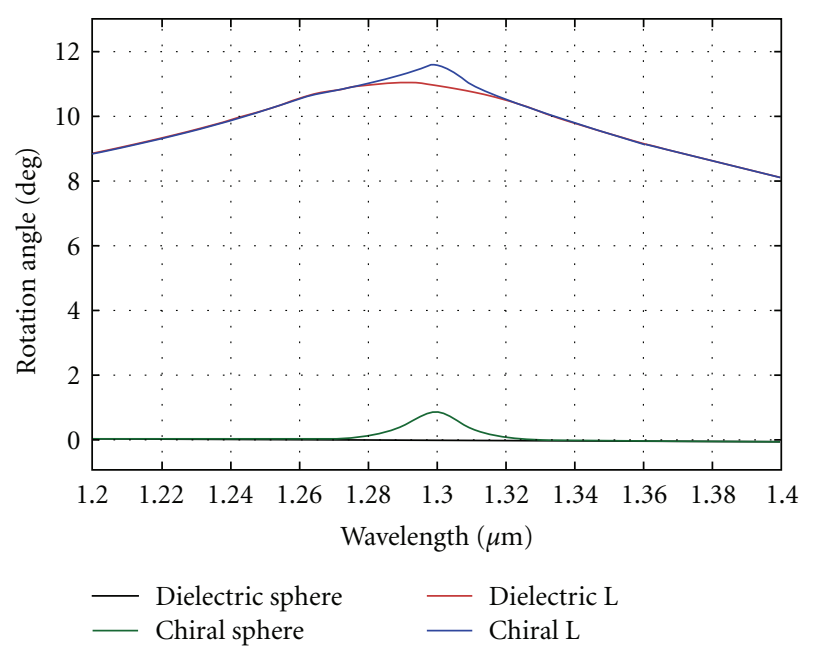

Figure 7: Predictions of the optical rotation angle for structures with and without molecular chirality. their respective spectra. This approach is demonstrated via the fabrication of a metamaterial consisting of a twodimensional array of chiral polymer-based planar chiral Lstructures. The fabrication process is described and modeling is performed to demonstrate the effects of multiscale chirality of the optical response (optical rotation) of such media. This new approach to metamaterials holds promise for the development of tunable, polymer-based negative index optical metamaterials with low loss.

\section{References}

[1] J. B. Pendry, "Negative refraction makes a perfect lens," Physical Review Letters, vol. 85, no. 18, pp. 3966-3969, 2000.

[2] R. A. Shelby, D. R. Smith, and S. Schultz, "Experimental verification of a negative index of refraction," Science, vol. 292, no. 5514, pp. 77-79, 2001.

[3] V. G. Veselago, "The electrodynamics of substance with simultaneously negative values of $\varepsilon$ and $\mu$," Soviet Physics Uspekhi, vol. 10, pp. 509-514, 1968. 
[4] Z. Jacob, L. V. Alekseyev, and E. Narimanov, "Optical hyperlens: far-field imaging beyond the diffraction limit," Optics Express, vol. 14, no. 18, pp. 8247-8256, 2006.

[5] Z. Liu, H. Lee, Y. Xiong, C. Sun, and X. Zhang, "Far-field optical hyperlens magnifying sub-diffraction-limited objects," Science, vol. 315, no. 5819, p. 1686, 2007.

[6] I. I. Smolyaninov, Y. J. Hung, and C. C. Davis, "Magnifying superlens in the visible frequency range," Science, vol. 315, no. 5819, pp. 1699-1701, 2007.

[7] Q. Bai, C. Liu, J. Chen, C. Cheng, M. Kang, and H. T. Wang, "Tunable slow light in semiconductor metamaterial in a broad terahertz regime," Journal of Applied Physics, vol. 107, no. 9, Article ID 093104, 8 pages, 2010.

[8] R. Singh, C. Rockstuhl, F. Lederer, and W. Zhang, "Coupling between a dark and a bright eigenmode in a terahertz metamaterial," Physical Review B, vol. 79, no. 8, Article ID 085111, 4 pages, 2009.

[9] S. Y. Chiam, R. Singh, C. Rockstuhl, F. Lederer, W. Zhang, and A. A. Bettiol, "Analogue of electromagnetically induced transparency in a terahertz metamaterial," Physical Review B, vol. 80, no. 15, Article ID 153103, 4 pages, 2009.

[10] J. B. Pendry, D. Schurig, and D. R. Smith, "Controlling electromagnetic fields," Science, vol. 312, no. 5781, pp. 1780-1782, 2006.

[11] D. Schurig, J. J. Mock, B. J. Justice et al., "Metamaterial electromagnetic cloak at microwave frequencies," Science, vol. 314, no. 5801, pp. 977-980, 2006.

[12] W. Cai, U. K. Chettiar, A. V. Kildishev, and V. M. Shalaev, "Optical cloaking with metamaterials," Nature Photonics, vol. 1, no. 4, pp. 224-227, 2007.

[13] N. Fang, H. Lee, C. Sun, and X. Zhang, "Sub-diffraction-limited optical imaging with a silver superlens," Science, vol. 308, no. 5721, pp. 534-537, 2005.

[14] M. Wuttig and N. Yamada, "Phase-change materials for rewriteable data storage," Nature Materials, vol. 6, no. 11, pp. 824-832, 2007.

[15] H. Kind, H. Yan, B. Messer, M. Law, and P. Yang, "Nanowire ultraviolet photodetectors and optical switches," Advanced Materials, vol. 14, pp. 158-160, 2002.

[16] W. M. Zhu, A. Q. Liu, X. M. Zhang et al., "Switchable magnetic metamaterials using micromachining processes," Advanced Materials, vol. 23, no. 15, pp. 1792-1796, 2011.

[17] N. Liu, H. Guo, L. Fu, S. Kaiser, H. Schweizer, and H. Giessen, "Three-dimensional photonic metamaterials at optical frequencies," Nature Materials, vol. 7, no. 1, pp. 31-37, 2008.

[18] J. Valentine, S. Zhang, T. Zentgraf et al., "Three-dimensional optical metamaterial with a negative refractive index," Nature, vol. 455, no. 7211, pp. 376-379, 2008.

[19] V. M. Shalaev, W. Cai, U. K. Chettiar et al., "Negative index of refraction in optical metamaterials," Optics Letters, vol. 30, no. 24, pp. 3356-3358, 2005.

[20] A. N. Grigorenko, A. K. Geim, H. F. Gleeson et al., "Nanofabricated media with negative permeability at visible frequencies," Nature, vol. 438, no. 7066, pp. 335-338, 2005.

[21] G. Dolling, C. Enkrich, M. Wegener, C. M. Soukoulis, and S. Linden, "Low-loss negative-index metamaterial at telecommunication wavelengths," Optics Letters, vol. 31, no. 12, pp. 1800-1802, 2006.

[22] G. Dolling, C. Enkrich, M. Wegener, C. M. Soukoulis, and S. Linden, "Simultaneous negative phase and group velocity of light in a metamaterial," Science, vol. 312, no. 5775, pp. 892$894,2006$.
[23] A. Boltasseva and V. M. Shalaev, "Fabrication of optical negative-index metamaterials: recent advances and outlook," Metamaterials, vol. 2, no. 1, pp. 1-17, 2008.

[24] H. S. Oh, S. Liu, H. S. Jee, A. Baev, M. T. Swihart, and P. N. Prasad, "Chiral poly(fluorene-alt-benzothiadiazole) (PFBT) and nanocomposites with gold nanoparticles: plasmonically and structurally enhanced chirality," Journal of the American Chemical Society, vol. 132, no. 49, pp. 17346-17348, 2010.

[25] S. Shukla, E. P. Furlani, X. Vidal, M. T. Swihart, and P. N. Prasad, "Subwavelength direct laser patterning of conductive gold nanostructures by simultaneous photopolymerization and photoreduction," ACS Nano, vol. 5, no. 3, pp. 1947-1957, 2011.

[26] S. Shukla, E. P. Furlani, X. Vidal, M. T. Swihart, and P. N. Prasad, "Two-photon lithography of sub-wavelength metallic structures in a polymer matrix," Advanced Materials, vol. 22, no. 33, pp. 3695-3699, 2010.

[27] A. Papakostas, A. Potts, D. M. Bagnall, S. L. Prosvirnin, H. J. Coles, and N. I. Zheludev, "Optical manifestations of planar chirality," Physical Review Letters, vol. 90, no. 10, Article ID 107404, 4 pages, 2003.

[28] M. Kuwata-Gonokami, N. Saito, Y. Ino et al., "Giant optical activity in quasi-two-dimensional planar nanostructures," Physical Review Letters, vol. 95, no. 22, Article ID 227401, 4 pages, 2005.

[29] A. Baev, M. Samoc, P. N. Prasad, M. Krykunov, and J. Autschbach, "A quantum chemical approach to the design of chiral negative index materials," Optics Express, vol. 15, no. 9, pp. 5730-5741, 2007.

[30] L. D. Landau, E. M. Lifshitz, and L. P. Pitaevskii, Electrodynamics of Continuous Media, Pergamon Press.

[31] E. P. Furlani and A. Baev, "Optical nanotrapping using cloaking metamaterial," Physical Review E, vol. 79, no. 2, Article ID 026607, 6 pages, 2009.

[32] E. P. Furlani and A. Baev, "Free-space excitation of resonant cavities formed from cloaking metamaterial," Journal of Modern Optics, vol. 56, no. 4, pp. 523-529, 2009.

[33] E. P. Furlani, R. Biswas, A. N. Cartwright, and N. M. Litchinitser, "Antiresonant guiding optofluidic biosensor," Optics Communications, vol. 284, no. 16-17, pp. 4094-4098, 2011.

[34] H. S. Oh, W.-Ch. Law, A. Baev et al., "A new twist on optical metamaterials: resonantly coupled hybrid chiral nanocomposites for metaphotonics," Nature Photonics. In press.

[35] H. S. Oh, H. S. Jee, A. Baev, M. T. Swihart, and P. N. Prasad, "Dramatic structural enhancement of chirality in photopatternable nanocomposites of chiral poly(fluorenealt-benzothiadiazole) (PFBT) in achiral SU-8 photoresist," Advanced Functional Materials. In press. 

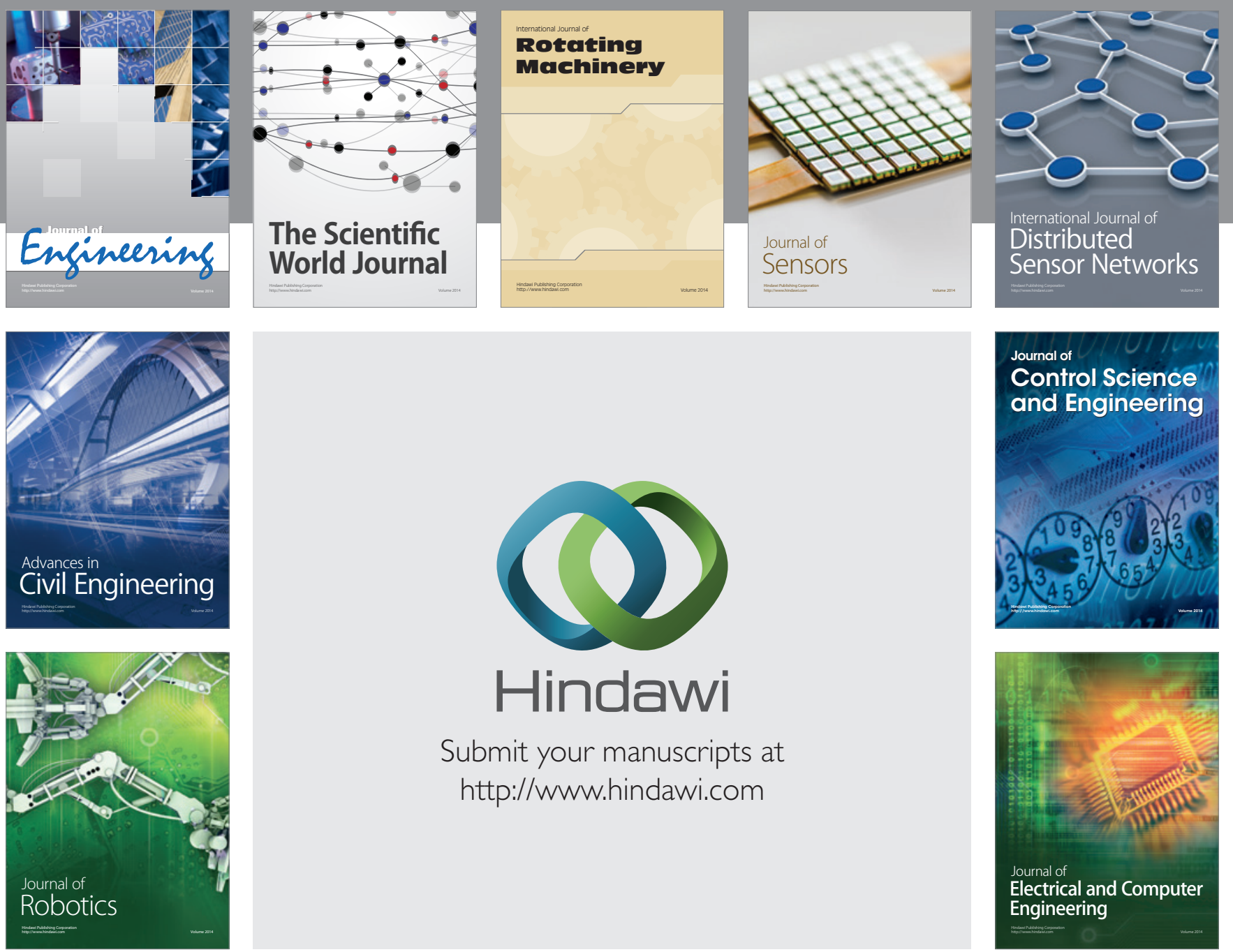

Submit your manuscripts at

http://www.hindawi.com
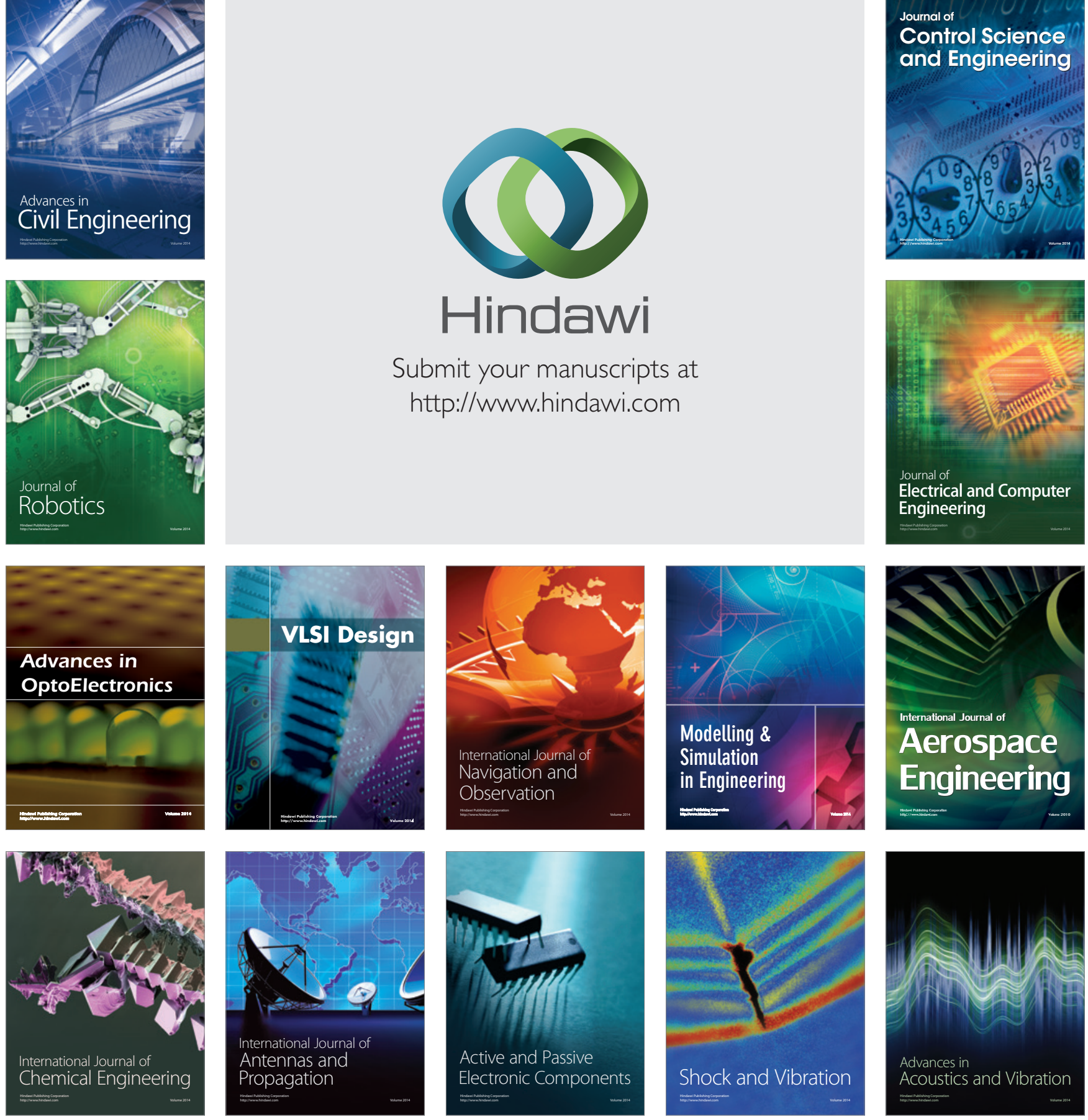